\title{
Possible adaptogenic effects of Momordica charantia on high-intensity training-induced alteration in the hypothalamic-pituitary-adrenal axis
}

\author{
Jang Soo Yook, ${ }^{1,+}$ Jae-Jun Kwak, ${ }^{2,+}$ Woo-Min Jeong, ${ }^{3}$ Young Hoon Song, ${ }^{4}$ Yasuaki Hijioka, ${ }^{5}$ Yasushi Honda, ${ }^{6}$ \\ Satbyul Estella Kim, ${ }^{5,6, *}$ and Min-Seong $\mathrm{Ha}^{7,8,9, *}$ \\ ${ }^{1}$ Center for Functional Connectomics, Brain Research Institute, Korea Institute of Science and Technology (KIST), \\ Hwarang-ro 14-gil 5, Seongbuk-gu, Seoul 02792, Republic of Korea \\ ${ }^{2}$ Department of National Defense Technology, Woosuk University, Daehak-ro 66, Jincheon-eup, Jincheon-gun, Chungcheongbuk-do 27841, Republic of Korea \\ ${ }^{3}$ WellCare Korea Co. Ltd., Wadong-ro 26, Danwon-gu, Ansan-si, Gyeonggi-do 15265, Republic of Korea \\ ${ }^{4}$ Department of Physical Education, Seoul National University, Gwanak-ro 1, Gwanak-gu, Seoul 08826, Korea \\ ${ }^{5}$ Center for Climate Change Adaption, National Institute for Environmental Studies, 16-2 Onogawa, Tsukuba, Ibaraki 305-8506, Japan \\ ${ }^{6}$ Faculty of Health and Sport Sciences, University of Tsukuba, 1-1-1 Tennoudai, Tsukuba, Ibaraki 305-8574, Japan \\ ${ }^{7}$ Department of Sports Neuroscience, Advanced Research Initiative for Human High Performance (ARIHHP) and ${ }^{8}$ Laboratory of Exercise Biochemistry and \\ Neuroendocrinology, Faculty of Health and Sports Sciences, University of Tsukuba, 1-1-1 Tennoudai, Tsukuba, Ibaraki 305-8574, Japan \\ ${ }^{9}$ Sport Culture Science Department, Dongguk University-Seoul, 30, Pildong-ro 1-gil, Jung-gu, Seoul 04620, Republic of Korea
}

This study investigated the effects of a drink supplement containing Momordica charantia extract from bitter melon on physical fitness and levels of stress hormones during a four-week exercise training program in a hot environment. Ten male tennis players were orally administrated in a four-week $(100 \mathrm{ml}, 6$ times a day), and the pre- and post-supplementation levels of different physical fitness variables and cortisol, and adrenocorticotropic hormone in plasma were measured at four time-points-before (baseline), during, and after the exercise, and on the next day of the supplementation. The findings showed that the supplementation has significant positive effects on enhancement of physical fitness parameters especially balance $(d=22.10, p=0.013)$, flexibility $(d=4.83, p=0.015)$, and cardiorespiratory fitness $(d=10.00$, $p=0.030$ ). Moreover, the adrenocorticotropic hormone levels were reduced during the exercise, and the cortisol levels showed the decreasing trend during and after the exercise, which was correlated with the change of cardiorespiratory fitness $(r=0.65$, $p<0.05)$. These results indicated the possible adaptogenic effects of Momordica charantia extract intake. Based on the findings, we suggest that Momordica charantia could be used as a source of adaptogenic supplement to alleviate the exercise- and environmentinduced stress.

Key Words: Momordica charantia, stress hormone, HPA axis, high-intensity exercise, adaptogenic effects

ntermittently, athletes undergo intense training with overload or in a strenuous environmental condition such as high temperature to improve their performance. However, excessive training with an inadequate recovery strategy can lead to an inhabitation in the beneficial consequences of exercise with fatigue, which is referred to as overreaching and overtraining. ${ }^{(1)}$ Such stressful conditions lead to alteration of several endocrine systems, notably the activation of the hypothalamic-pituitaryadrenal (HPA) axis. ${ }^{(2)}$ Accordingly, a well-designed training program with appropriate nutritional intervention is required, not only to prevent the physiological effects of stress but also to improve the exercise performance in the days following a highintensity exercise under extreme environments. ${ }^{(3)}$

A central system of the stress response is the HPA axis, regulation of which plays a major role in intense exercise-related physiological homeostasis. ${ }^{(4)}$ In response to stressful exercise, corticotropin-releasing hormone $(\mathrm{CRH})$ that is secreted by the hypothalamus acts on the anterior pituitary for releasing adrenocorticotropic hormone $(\mathrm{ACTH})$, which in turn stimulates the adrenal cortex to secrete cortisol. ${ }^{(5)}$ Alternation of HPA axis activities in physical exercise-trained athletes has been abundantly reported. In trained male athletes, a prolonged submaximal exercise has been reported to elevate the levels of cortisol and ACTH in the plasma. ${ }^{(6)}$ In addition, impaired regulation of HPA axis activity in response to exhaustive exercise-induced stress has also been observed in overstrained athletes. ${ }^{(7,8)}$ These studies suggest that the alteration of the HPA axis activity in highly trained athletes could have relevance for evaluating their overtraining status and improvement of sports performance.

Natural plant-derived phytochemicals induce an adaptive response of stress along with the activation of HPA axis. ${ }^{(9)}$ In addition, there are growing interests in the effects of phytochemicals that appears to have positive effects on exerciseinduced oxidative stress for improving the adaptation responses to exercise training. ${ }^{(10)}$ These are considered as adaptogens (antistress) that stabilize the physiologic stress responses through enhancement of tolerance to various stressors, including physical exercise, and have been suggested as the promising metabolic regulators on sports performance. ${ }^{(1)}$ Of the several plants used for extracting the phytochemicals, Momordica charantia, also known as bitter melon or bitter gourd, a member of the Cucurbitaceae family containing various phytochemicals, such as vitamins amino acids, essential oils, minerals, phenolic acids, alkaloids, flavonoids, quinines, saponins, and triterpenoids, is a valuable resource. ${ }^{(12)}$

†JS Yook and J-J Kwak contributed equally to this work.

*To whom correspondence should be addressed.

E-mail: haminseong@dgu.ac.kr (MSH);

kim.estells.ge@u.tsukuba.ac.jp (SEK) 


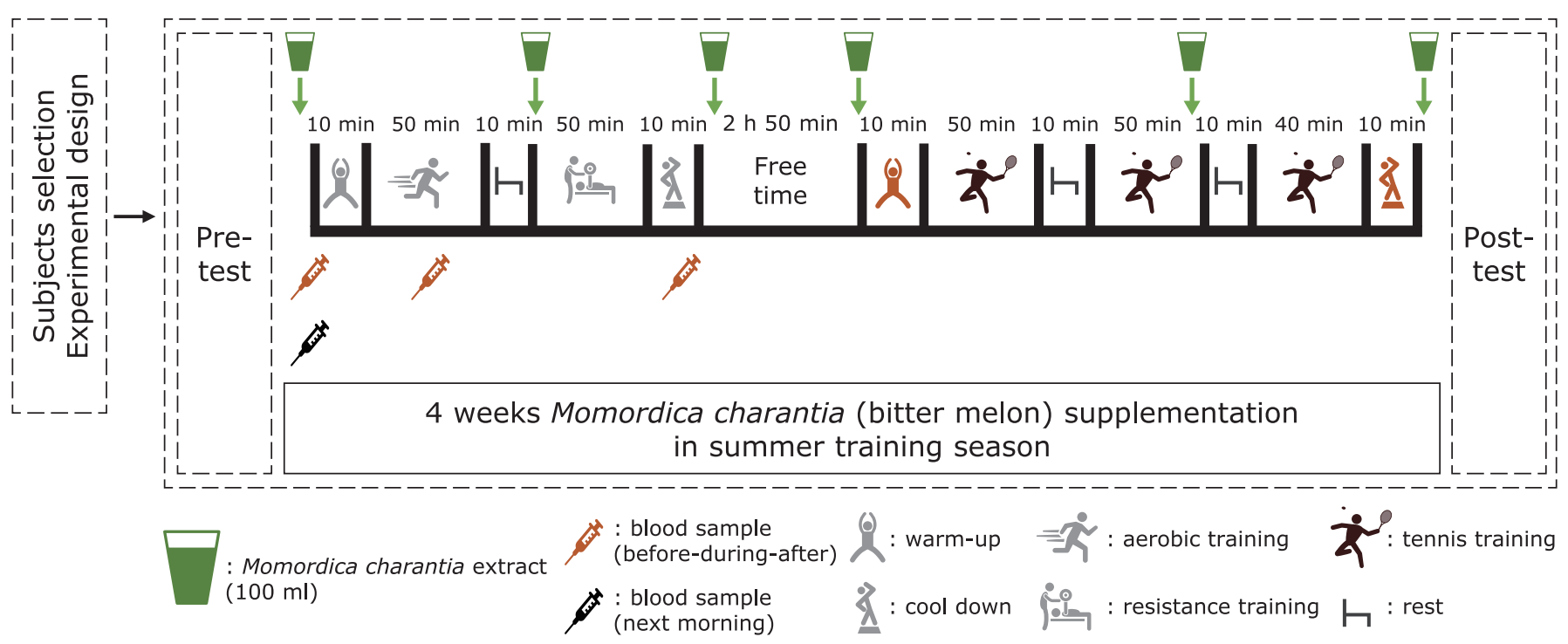

Fig. 1. Study design to investigate the effects of Momordica charantia extract (MCE) supplementation on physical fitness and stress hormones (ACTH and cortisol) in tennis players during a four-week high-intensity training period. See color figure in the on-line version.

M. charantia exhibits various biological activities, including antioxidant, antidiabetic, anticancer, and anti-inflammatory properties. ${ }^{(13)}$ Previous studies using experimental animals have shown that $M$. charanitia possesses adaptogenic activity under acute and chronic stress ${ }^{(14)}$ as well as physically stressful conditions. ${ }^{(15)}$ However, its protective effects on heavy physical training-induced stress have not been explored fully.

As previously noted, $M$. charanitia showed anti-stress effects in animal studies. Recently, we found that $M$. charanitia extract (MCE) supplementation induced anti-fatigue effects on highintensity trained athletes, resulting in significant regulation in central fatigue-related hormone release, ${ }^{(16)}$ which is warranted to further investigate its influence on stress-related hormonal response, particularly in the circulating plasma levels of ACTH and cortisol.

Therefore, in the present study, we aimed to investigate the effects of MCE supplementation on the physical fitness and alteration of HPA axis parameters in highly trained tennis players trained for four weeks in a high-temperature environment.

\section{Materials and Methods}

Participants. We selected 10 male tennis players (average age: $22.2 \pm 0.9$, average height: $177.2 \pm 6.7$, average weight: $70.8 \pm 12.3$, average careers: $5.9 \pm 2.7$ ) from Pusan National University. The selected players participated in all training programs and matches and did not perform any personal training other than team training. The participants did not have any injuries or neurological or musculoskeletal diseases, no history of hormone replacement therapy, drug or alcohol abuse, smoking, allergies, chronic diseases, thyroid disease, eating disorders, and consumption of any supplement that could affect the experiment; all parameters that could affect the experiment were controlled by doctor examinations/interviews and physical examinations. Before fully informed of the research intention and purpose, and the consent forms were obtained from all participants. The Institutional Human Research Committee of the Pusan National University approved the research proposal, which followed the Declaration of Helsinki Declaration and Ethical Principles.

Study design. This four-week study was conducted as an intervention study design to investigate the impact of MCE intake on physical fitness and stress hormones in 10 male university tennis players after intensive training (Fig. 1). The four-week training was conducted in a hot environment condition with a daily average temperature of $32.12 \pm 2.20^{\circ} \mathrm{C}$, the sensible temperature of $33.53 \pm 1.91^{\circ} \mathrm{C}$, and temperature-humidity index (THI) of $80.13 \pm 1.25$. Three doses of MCE were administered, with one dose $(100 \mathrm{ml})$ each before, during, and after the exercise, both in the morning and afternoon totaling to $600 \mathrm{ml}$ of MCE per day. The participants underwent a high-intensity exercise regime, and the span of the exercise was for $100 \mathrm{~min}$ in the morning (aerobic and resistance) and $140 \mathrm{~min}$ in the afternoon (tennis training), five days per week, for a total of 4 weeks, intending to improve physical condition and technique. Eight physical fitness variables, including flexibility, agility, power, muscle strength, muscular endurance, reaction function, cardiorespiratory fitness, and balance, and the levels of stress hormones, including cortisol and $\mathrm{ACTH}$ were measured before, during, and after exercise and in the morning following the day of exercise before consumption of MCE (week 0) and after consumption of MCE regularly, till the end of the training period. For the successful completion of the study, the researchers monitored, not only the training program but also the subjects' lifestyle (camp accommodation). In addition, considering the continuous exposure to the high-temperature environment due to the characteristics of the study, heatstroke, heat edema, heat exhaustion, heat cramping, heat rash) and nutritional status was repeatedly checked until the end of the study.

Procedures. M. charantia containing biologically active compounds such as polypeptides, saponins, alkaloids, flavonoids, phenolic acids, and carotenoids produced in Korea were used in this study. ${ }^{(17,18)}$ Then the MCE was prepared following the method optimized for natural plants as described in the previous studies. ${ }^{(19,20)}$ Subsequently, $4 \mathrm{~kg}$ of dried MCE and $6,000 \mathrm{ml}$ of water was added to the extractor, and the mixture was extracted by heating at $100^{\circ} \mathrm{C}$ under a pressure of $0.7 \mathrm{~kg} / \mathrm{cm}^{2}$ for $3 \mathrm{~h}$. The extracted MCE was then sealed in a small plastic bag of $100 \mathrm{ml}$ and stored in a refrigerator. To analyze the basic ingredients, we commissioned the Korea Food Research Institute and Pukyong National University Food Analysis Center. The main ingredients of MCE were water $(82.68 \mathrm{~g})$, sodium $(10.40 \mathrm{~g})$, vitamin C $(6.42 \mathrm{~g})$, calorie $(0.40 \mathrm{~g})$, and carbohydrate $(0.10 \mathrm{~g})$.

Training program. The training was performed following the revised protocol reported by Kwak et al. ${ }^{(21)}$ Briefly, the participants were trained $100 \mathrm{~min}$ in the morning (aerobic and 
resistance) and $140 \mathrm{~min}$ in the afternoon (tennis training) per day, five days a week for a total of four weeks. During the training period, the subjects performed body conditioning, professional skill training, aerobic training, and resistance training. The morning training program consisted of a 10 min warm-up of 40$60 \%$ HRR (RPE 12-14) and a 10 min cool-down of $20-40 \%$ HRR (RPE 9-11) and a 100 min of the main training comprising $50 \mathrm{~min}$ aerobic exercise with 70-90\% HRR (RPE 15-19) intensity and 50 min resistance training with individual loads equivalent to 60 $85 \%$ 1RM (OMNI RES 6-8). The afternoon training included 100 -min of professional skill-related training and 40 min single match with 70-90\% HRR (RPE 15-19) intensity. A 10 min rest in between the main training was followed both in the morning and afternoon.

Statistical analysis. The required number of sample size was calculated using the G-power ver. 3.1 Windows program (Kiel University, Kiel, Germany), based on an effect size of a 0.25-point (default), an alpha level of 0.05 , and $30 \%$ power. $^{(22,23)}$ The results indicated that 10 participants were needed; assuming a dropout rate of $20 \%$, the sample size was set to 8 participants. All data were expressed as mean \pm SD. To compare the mean differences between physical fitness variables before and after drinking MCE, variables with normally distributed data (based on the Shapiro test: $p>0.05$ ) were analyzed using the paired $t$ test; variables with non-normally distributed data (Shapiro test: $p<0.05)$ were analyzed using the Wilcoxon signed-rank test. Two-way mixed ANOVA was applied to find the effect of MCE on stress hormone level and exercise. To compare the level of heat stress hormones before and after MCE-supplementation for each measurement period (baseline, during exercise, after exercise, and the next day), stratified Wilcoxon signed-rank tests were performed. We further analyzed using Cousineau-Morey Corrected error bars to remove the individual differences in changes in heat stress hormones concentration at each measurement period. Correlation analysis was performed following Pearson's correlation coefficients to estimate the relationship between the changes in physical fitness variables and stress hormones before and after the intake of MCE. All Statistical significance was set at $p<0.05$. All analyses were performed with the statistical software R ver. 3.6.1 (R Foundation for Statistical Computing, Vienna, Austria).

\section{Results}

Effect of MCE on levels of the physical fitness variables. We investigated the effects of MCE supplementation on sports performance-related physical fitness variables, which were applied before and after the intervention (Table 1). Of the eight variables measured, the mean values of three variables, including balance $(d=22.10, p=0.013)$, flexibility $(d=4.83, p=0.015)$, and cardiorespiratory fitness $(d=10.00, p=0.030)$ were significantly different between the two interventions.

Effect of MCE on the levels of cortisol and ACTH. To investigate the influence of MCE supplementation on stress-related hormones, we measured cortisol and ACTH levels in plasma preand post-training program at four measurement periods-before (baseline), during, and after exercise, and the next day. The twoway mixed ANOVA showed a time effect in cortisol $(F=3.35$, $p=0.03)$ and a slight tendency of interaction in ACTH $(F=2.68$, $p=0.06$ ) (Table 2). When compared the levels of cortisol and $\mathrm{ACTH}$ at each measurement period, there were no significant differences (Fig. 2). However, significant differences in the levels of cortisol and ACTH (Supplemental Fig. 1*) were observed at individual participant levels. By normalizing the sample variance through Cousineau-Morey methods, ${ }^{(24)}$ we reanalyzed the changes in the levels of cortisol and ACTH after removing the individual differences. As shown in Fig. 3, there was a different pattern of cortisol and ACTH levels in the pre- and post-test of MCE supplementation, while the post-test showed a lowering effect compared to pre-test in both hormone levels. The post-test cortisol levels of MCE supplementation were lower during and after the exercise period than the pre-test (Fig. 3A). However, the post-test ACTH levels of MCE supplementation were lower only during the exercise period as compared to the pre-test levels (Fig. 3B). Moreover, both the post-test cortisol and ACTH levels were nonsignificantly higher on the next day than the pre-test levels.

Table 1. The estimated differences between the mean values of the eight physical performance variables before and after MCE supplementation

\begin{tabular}{|c|c|c|c|c|c|c|}
\hline \multirow{2}{*}{ Variable } & \multirow{2}{*}{$\begin{array}{l}\text { Mean difference } \\
\text { (post-pre) }\end{array}$} & \multirow{2}{*}{$\begin{array}{c}\text { Shapiro-normality test } \\
p \text { value }\end{array}$} & \multicolumn{2}{|c|}{ Paired $t$ test } & \multicolumn{2}{|c|}{ Wilcoxon signed-rank test } \\
\hline & & & $t$ value & $p$ value & V & $p$ value \\
\hline Flexibility & 4.83 & 0.075 & -2.99 & $0.015^{*}$ & 2 & $0.006 * *$ \\
\hline Agility & -0.2 & $0.001 * * *$ & 0.07 & 0.945 & 11.5 & 0.395 \\
\hline Power & 2 & 0.093 & -1.86 & 0.096 & 8 & 0.181 \\
\hline Muscular strength & 0.28 & 0.244 & -0.71 & 0.497 & 23 & 0.683 \\
\hline Muscular endurance & 2.2 & $0.009 * *$ & -2.34 & $0.044^{*}$ & 0 & 0.058 \\
\hline Reaction function & 18 & 0.32 & -1.59 & 0.147 & 13 & 0.16 \\
\hline Cardiorespiratory fitness & 10 & 0.645 & -2.57 & $0.030 *$ & 5 & $0.044^{*}$ \\
\hline Balance & 22.1 & $0.006 * *$ & -2.91 & $0.017^{*}$ & 1 & $0.013^{*}$ \\
\hline
\end{tabular}

${ }^{*} p<0.05,{ }^{* *} p<0.01, * * * p<0.001$.

Table 2. Two-way (Group $\times$ Time) analysis of variance of the levels of cortisol and ACTH

\begin{tabular}{lcccccc}
\hline Source & & Sum of Squares & Df & Error SS & $F$ & $p$ value \\
\hline Cortisol & Group & 21 & 1 & $1,836.4$ & 0.21 & 0.66 \\
& Time & 190 & 3 & $1,020.6$ & 335 & $0.03^{*}$ \\
& Group $\times$ Time & 70 & 3 & $1,020.6$ & 1.23 & 0.31 \\
\hline ACTH & Group & 2,153 & 1 & 35,071 & 1.1 & 0.31 \\
& Time & 765 & 3 & 32,905 & 0.42 & 0.74 \\
& Group $\times$ Time & 4,904 & 3 & 32,905 & 268 & $0.06^{*}$ \\
\hline
\end{tabular}

${ }^{\star} p<0.05$. 

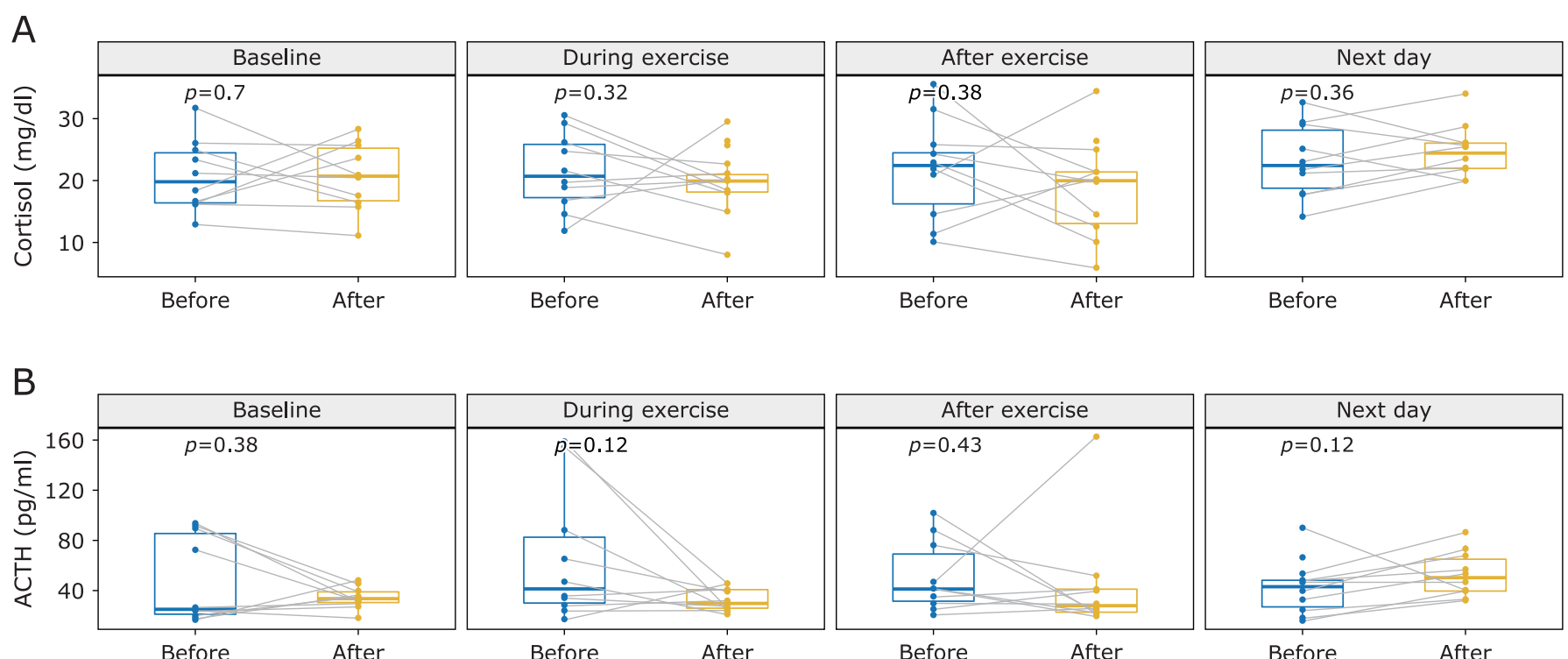

Fig. 2. Comparison of the levels of the stress hormones (A) cortisol and (B) ACTH before and after the MCE supplementation. The levels of the hormones were estimated at four time-points-before, during, and after the exercise, and the day following the exercise day. Colored dots indicate the values obtained for an individual subject $(n=10)$. See color figure in the on-line version.
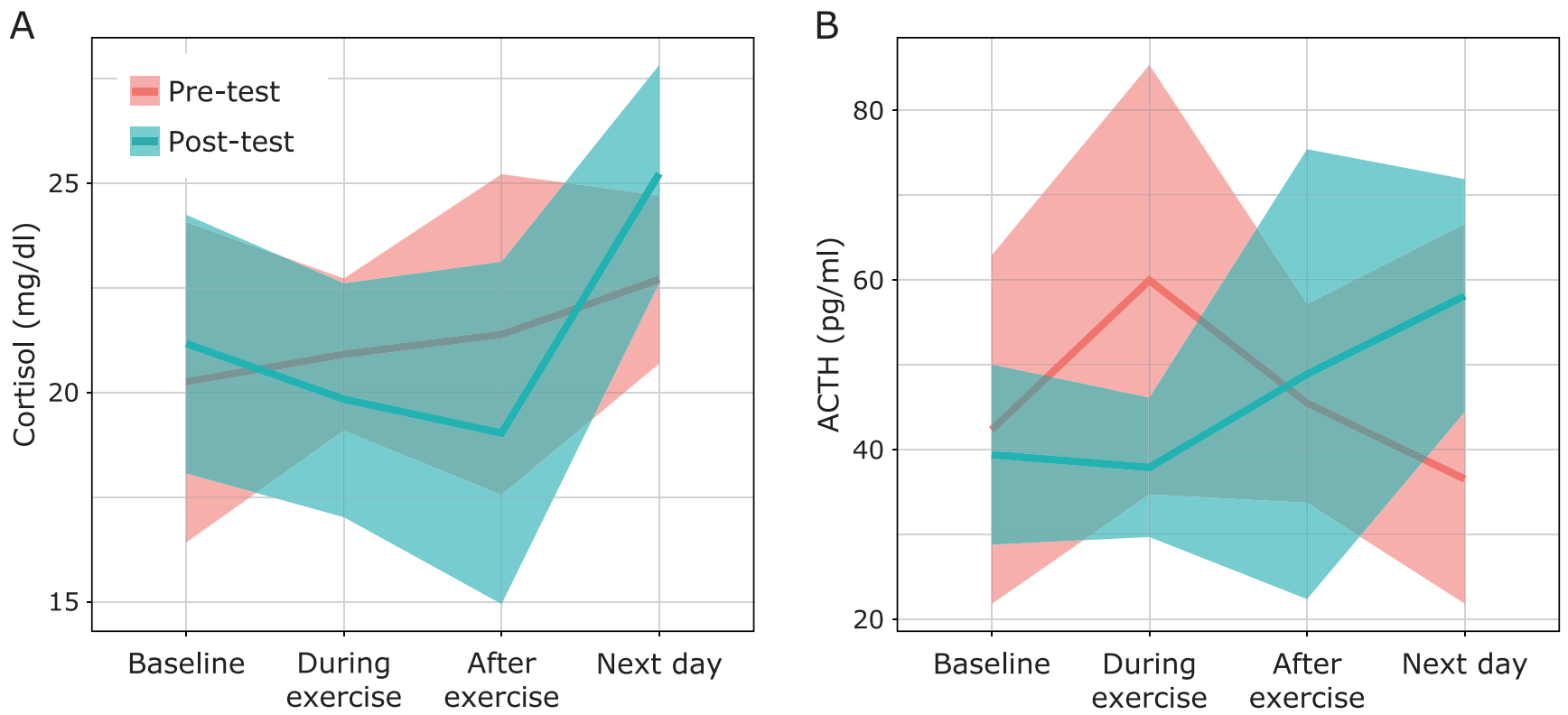

Fig. 3. Comparison of the levels of the stress hormones after normalizing the individual sample variance through Cousineau-Morey method (A) cortisol and (B) ACTH, pre- and post-supplementation of MCE. The levels of the hormones were estimated at four time-points-before (baseline), during, and after the exercise, and the day following the exercise day (next day). Error bars are within-subject standard errors. Pre- and post-test effects are indicated in light blue line and orange line, respectively. See color figure in the on-line version.

Correlation between the physical fitness variables and the levels of stress hormones. To determine whether the change of stress hormones was related to the intensive trainingaffected exercise performance with the MCE supplementation, we analyzed the correlation between the delta $(\Delta$; the difference between post- and pre-tests) values of physical fitness variables and individual cortisol and ACTH changes (Fig. 4). There was a significant correlation between the change of cortisol and ACTH before and after intake of MCE $(r=0.88, p<0.05)$. The change in the post- and pre-test cortisol levels was significantly correlated with the change in the level of cardiorespiratory fitness before and after intake of MCE $(r=0.65, p<0.05)$. However, there was no significant correlation between the change in ACTH level and the changes in the variables of physical fitness.

\section{Discussion}

Stress management is important for improving the athlete's performance in sports competitions as well as intensive training camps. According to this perspective, we investigated whether 


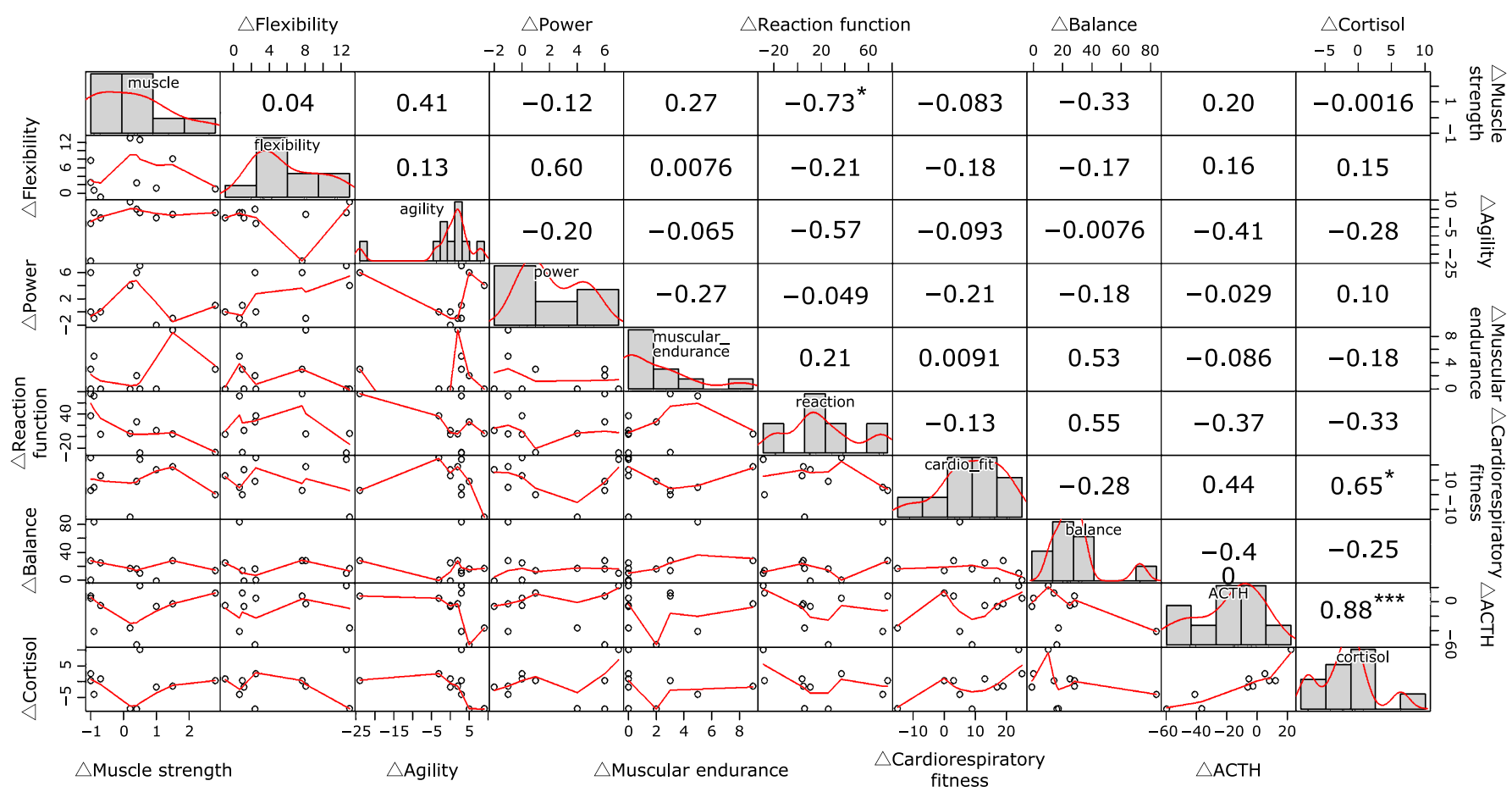

Fig. 4. Correlation between the changes $(\Delta)$ in the physical fitness variables and the levels of cortisol and ACTH pre- and post-test of MCE supplementation. Correlation analysis was performed following Pearson's correlation coefficients. Each circle indicates an individual subject $(n=10)$. ${ }^{*} p<0.05, * * * p<0.001$. See color figure in the on-line version.

MCE supplementation can induce anti-stress effects, through assessment of cortisol and ACTH levels in plasma, during the four weeks of high-intensity training camp in a high-temperature environment. In this study, we found that the tennis players who consumed the MCE showed significant enhancement of physical fitness levels. The decreasing trend of cortisol and ACTH levels during exercise were observed after the MCE supplementation. Our results indicate that the MCE might possess a potent role of adaptogenic effects through changing the HPA axis activity response to strenuous physical training in a hot environment.

Generally, athletes undergo intense training programs to maximize sports skill-related physical performance before the competitive period. ${ }^{(25)}$ The competitive tennis play that is characterized by the unpredictability of match duration, shot selection, and weather requires a solid combination of several physical abilities (i.e., cardiorespiratory response, strength, agility, flexibility, and power). ${ }^{(26,27)}$ Athletes exposed to stressful conditions, including strenuous exercise training, might inflict a condition of physiological fatigue in which these stress factors could adversely affect their physical performance. Recently, we have shown that the MCE supplementation induces positive effects on fatiguerelated hormones in response to a high-temperature environment combined with high-intensity training. ${ }^{(16)}$ In this study, a significant enhancement of flexibility, cardiorespiratory function, and balance in pre-post design paired test of MCE supplementation were observed in the subjects that included elite tennis players (Table 1), suggesting that MCE supplementation could benefit a diverse range of physical fitness and adequate recovery through its anti-fatigue effects.

Prolonged strenuous exercise in a hot environment exerts stress on the whole-body induces the release of hormone based on the HPA axis activity, which is dependent on the intensity or duration of the exercise. ${ }^{(28,29)}$ As physical exercise and heat are well known to be promising stress stimulators, the levels of cortisol and $\mathrm{ACTH}$, as a neuroendocrine response in HPA axis activity, are used as the potential biomarkers for monitoring the exerciseinduced stress as well as overtraining. ${ }^{(30,31)}$ In this study, we showed that the MCE supplementation did not induce a significant effect on the changes of both cortisol and ACTH levels at all measurement periods (Fig. 2), which could be due to the large variance observed at the individual subject levels. However, after removing the individual difference, the post-test levels of cortisol and ACTH were reduced (Fig. 3), which indicated that the MCE supplementation could have beneficial adaptogenic effects by counteracting HPA axis activity in athletes exposed to highintensity training under a hot environment.

There are several lines of evidence that support the novel function of the MCE in the exercise-related stress response. For instance, vitamin $\mathrm{C}$, a major content of the MCE, can lead a significant reduction of circulating cortisol in athletes, who underwent prolonged exercise, ${ }^{(32,33)}$ which is consistent with our study that has shown that the MCE supplementation can attenuate the increase in plasma cortisol levels in response to high-intensity exercise. Furthermore, oral administration of magnesium, a micronutrient derived from the MCE, in rugby players influenced the alternation of cortisol and ACTH levels on the day before and after a competitive match. ${ }^{(34)}$ Similarly, in this study, there was a trend toward lower levels of cortisol and ACTH during exercise after the intake of MCE (Fig. 3). Both stress hormone levels returned to the normal range in the next day. These trends implicated the changes in the levels of the stress hormones at various time-points during exercise and recovery following the high-intensity training.

As mentioned earlier, physical exercise can stimulate the HPA axis activity, resulting in alternation in the circulating levels of cortisol. As per the exercise physiology reference sources, moderate to the high intensity of exercise at or above $60 \%$ of maximal oxygen consumption $\left(\mathrm{VO}_{2 \max }\right)$ provoked a significant increase in cortisol levels, but the low intensity of exercise $(40 \%$ of $\left.\mathrm{VO}_{2 \max }\right)$ induced a reduction in cortisol levels. ${ }^{(28,35)}$ These 
previous studies suggest that there is a significant interaction between the influence of cardiovascular fitness and cortisol response from the HPA axis. In this study, we found that the enhancement of cardiovascular fitness by the four weeks of highintensity training corresponded to the change in circulating cortisol levels (Fig. 4), suggesting a potential mechanism of adaptogenic effects for the enhanced physical performance and recovery. Further investigations are suggested to be undertaken to conduct a chamber study controlling all other environmental factors with the control group.

As a limitation of this study, small sample size should be considered when interpreting and generalizing the results that the effectiveness of the MCE supplementation on adaptogenic activity in athletes. Only recruiting male subjects in the present study also limits applying the beneficial effects of the MCE in female athletes. Therefore, further research on the effects of the MCE intake in female athletes with large sample size is needed. Because phytochemicals exhibit potential benefits on neuronal activity and plasticity for enhancing brain function, ${ }^{(9,36)}$ further research investigating a novel role of MCE on cognitive function related with stress response is warranted.

In conclusion, our findings from this study demonstrated that a four-week supplementation of the MCE during the high-intensity training in a hot environment enhances physical fitness levels and induce a reduction of stress hormones. These results indicated that the MCE could help to minimize the adverse effects of stressful exercise and condition-induced performance decrements, thereby allowing the athletes to have effective training. Thus, the MCE could be a source of adaptogenic supplement with better tolerance of the exercise- and environment-induced stress.

\section{Author Contribution}

Conceptualization, J-JK SEK, and M-SH; methodology, J-JK; validation, $\mathrm{SEK}$, and $\mathrm{M}-\mathrm{SH}$; formal analysis, $\mathrm{SEK}$; investigation, J-JK, W-MJ, and YHS; resources, J-JK; data curation, SEK; writing - original draft preparation, JSY, and M-SH; writingreview and editing, SEK, YHijioka, M-SH, and SEK; visualization, JSY, and SEK; supervision, SEK, and MSH; medical expertise, YHonda.

\section{Acknowledgments}

The authors would like to thank the researchers for their expertise throughout the testing procedures and the players and staff at the Pusan National University Tennis team for their hard work and cooperation throughout the study. This work was partially supported by the Climate Change Adaptation Research Program of the National Institute for Environmental Studies, Japan. SEK was supported by the Basic Science Research Program through the National Research Foundation of Korea (2019R1A6A3A03031690).

\section{Conflicts of Interest}

No potential conflicts of interest were disclosed.

\section{References}

1 Budgett R. Fatigue and underperformance in athletes: the overtraining syndrome. Br J Sports Med 1998; 32: 107-110.

2 Angeli A, Minetto M, Dovio A, Paccotti P. The overtraining syndrome in athletes: a stress-related disorder. J Endocrinol Invest 2004; 27: 603-612.

3 Rawson ES, Miles MP, Larson-Meyer DE. Dietary supplements for health, adaptation, and recovery in athletes. Int J Sport Nutr Exerc Metab 2018; 28 : 188-199.

4 Morgan JA, Corrigan F, Baune BT. Effects of physical exercise on central nervous system functions: a review of brain region specific adaptations. $J \mathrm{Mol}$ Psychiatr 2015; 3: 3.

5 Viru A, Viru M. Cortisol-essential adaptation hormone in exercise. Int $J$ Sports Med 2004; 25: 461-464.

6 Inder WJ, Hellemans J, Swanney MP, Prickett TC, Donald RA. Prolonged exercise increases peripheral plasma ACTH, CRH, and AVP in male athletes. J Appl Physiol (1985) 1998; 85: 835-841.

7 Urhausen A, Gabriel HH, Kindermann W. Impaired pituitary hormonal response to exhaustive exercise in overtrained endurance athletes. Med Sci Sports Exerc 1998; 30: 407-414.

8 Brooks K, Carter J. Overtraining, exercise, and adrenal insufficiency. J Nov Physiother 2013; 3: 11717.

9 Fujii Y, Suzuki K, Adachi T, Taira S, Osakabe N. Corticotropin-releasing hormone is significantly upregulated in the mouse paraventricular nucleus following a single oral dose of cinnamtannin A2 as an (-)-epicatechin tetramer. J Clin Biochem Nutr 2019; 65: 29-33.

10 D'Angelo S. Polyphenols and athletic performance: a review on human data. In: Plant Physiological Aspects of Phenolic Compounds. IntechOpen 2019. DOI: 10.5772 intechopen.85031

11 Molinos Domene Á. Effects of adaptogen supplementation on sport performance. A recent review of published studies. J Hum Sport Exerc 2013; 8: 1054-1066.

12 Wang S, Li Z, Yang G, Ho CT, Li S. Momordica charantia: a popular healthpromoting vegetable with multifunctionality. Food Funct 2017; 8: 17491762.

13 Jia S, Shen M, Zhang F, Xie J. Recent advances in Momordica charantia: functional components and biological activities. Int J Mol Sci 2017; 18: 2555.

14 Kavitha N, Babu SM, Rao ME. Influence of Momordica charantia on oxidative stress-induced perturbations in brain monoamines and plasma corticosterone in albino rats. Indian J Pharmacol 2011; 43: 424-428.
15 Meera S, Nagarjuna CG. Antistress and immunomodulatory activity of aqueous extract of Momordica charantia. Pharmacogn Mag 2009; 5: 69-73.

16 Kwak J-J, Yook JS, Ha M-S. Potential biomarkers of peripheral and central fatigue in high-intensity trained athletes at high-temperature: a pilot study. $J$ Immunol Res 2020; 2020: 4768390.

17 Ahn MJ, Yuk HJ, Lee HY, et al. Effect of the enhanced biological activities and reduced bitter taste of Bitter melon (Momordica charantia L.) by roasting. $J$ Agric Life Sci 2015; 49: 107-119.

$18 \mathrm{Kim} \mathrm{M}-\mathrm{W}$. Effect of bitter melon on plasma blood glucose and cholesterol levels in streptozotocin induced diabetic rats. J East Asian Soc Diet Life 2013; 23: 704-712.

19 Ha MS, Kim JH, Ha SM, Kim YS, Kim DY. Positive influence of aqua exercise and burdock extract intake on fitness factors and vascular regulation substances in elderly. J Clin Biochem Nutr 2019; 64: 73-78.

20 Ha MS, Kim JH, Kim YS, Kim DY. Effects of aquarobic exercise and burdock intake on serum blood lipids and vascular elasticity in Korean elderly women. Exp Gerontol 2018; 101: 63-68.

21 Kwak JJ, Sung GD, Baek YH. Effects of herbal drinks intake for summer training physical fitness, $\mathrm{VO}_{2 \max }$ and $\mathrm{E}$ in male college tennis players. Teach Educ Res 2013; 52: 639-648.

22 Faul F, Erdfelder E, Lang AG, Buchner A. G*Power 3: a flexible statistical power analysis program for the social, behavioral, and biomedical sciences. Behav Res Methods 2007; 39: 175-191.

23 Cohen J. Statistical Power Analysis for the Behavioral Sciences (2nd ed.). Hillsdale, NJ: Lawrence Erlbaum Associates, Publishers, 1988.

24 Morey RD. Confidence intervals from normalized data: a correction to Cousineau (2005). Tutor Quant Methods Psychol 2008; 4: 61-64.

25 Buchheit M, Racinais S, Bilsborough JC, et al. Monitoring fitness, fatigue and running performance during a pre-season training camp in elite football players. J Sci Med Sport 2013; 16: 550-555.

26 Fernandez-Fernandez J, Ulbricht A, Ferrauti A. Fitness testing of tennis players: how valuable is it? Br J Sports Med 2014; 48 Suppl 1: i22-i31.

27 Kovacs MS. Applied physiology of tennis performance. Br J Sports Med 2006; 40: 381-386.

28 Hill EE, Zack E, Battaglini C, Viru M, Viru A, Hackney AC. Exercise and circulating cortisol levels: the intensity threshold effect. J Endocrinol Invest 2008; 31: 587-591.

29 Brenner I, Shek PN, Zamecnik J, Shephard RJ. Stress hormones and the 
immunological responses to heat and exercise. Int J Sports Med 1998; 19: 130-143.

30 Cadegiani FA, Kater CE. Hypothalamic-pituitary-adrenal (HPA) axis functioning in overtraining syndrome: findings from endocrine and metabolic responses on overtraining syndrome (EROS)_EROS-HPA axis. Sports Med Open 2017; 3: 45.

31 Urhausen A, Gabriel H, Kindermann W. Blood hormones as markers of training stress and overtraining. Sports Med 1995; 20: 251-276.

32 Peters EM, Anderson R, Nieman DC, Fickl H, Jogessar V. Vitamin C supplementation attenuates the increases in circulating cortisol, adrenaline and antiinflammatory polypeptides following ultramarathon running. Int $J$ Sports Med 2001; 22: 537-543.

33 Peters EM, Anderson R, Theron AJ. Attenuation of increase in circulating cortisol and enhancement of the acute phase protein response in vitamin Csupplemented ultramarathoners. Int J Sports Med 2001; 22: 120-126.
34 Dmitrašinović G, Pešić V, Stanić D, Plećaš-Solarović B, Dajak M, Ignjatović S. ACTH, cortisol and IL-6 levels in athletes following magnesium supplementation. J Med Biochem 2016; 35: 375-384.

35 Hackney AC. Stress and the neuroendocrine system: the role of exercise as a stressor and modifier of stress. Expert Rev Endocrinol Metab 2006; 1: 783792.

36 Yook JS, Lee M. Potential role of phytochemicals in brain plasticity: focus on polyunsaturated fatty acids. J Exerc Nutr Biochem 2020; 24: 14-18.

This is an open access article distributed under the terms of the Creative Commons Attribution-NonCommercial-NoDerivatives License (http://creativecommons.org/licenses/by-nc-nd/4.0/). 